\title{
Lipidomic analysis reveals a radiosensitizing role of gamma-linolenic acid in glioma cells
}

\author{
Otilia Antal a,1, Mária Péter b,1 ${ }^{\text {b }}$ László Hackler Jr. ' , Imola Mán ${ }^{\text {c }}$, Gábor Szebeni ${ }^{\text {, }}$, Ferhan Ayaydin ${ }^{\text {d, }}$ \\ Katalin Hideghéty ${ }^{\mathrm{e}}$, László Vigh ${ }^{\mathrm{b}}$, Klára Kitajka ${ }^{\mathrm{a}, \mathrm{c}}$, Gábor Balogh ${ }^{\mathrm{b}, 2}$, Laszló G Puskás ${ }^{\mathrm{a}, \mathrm{c}, *, 2}$ \\ a Laboratory for Functional Genomics, Institute of Genetics, Biological Research Center of the Hungarian Academy of Sciences, Szeged H-6726, Hungary

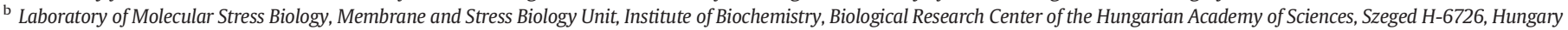 \\ c Avidin Ltd., Szeged H-6726, Hungary ${ }^{3}$ \\ d Cellular Imaging Laboratory, Biological Research Center of the Hungarian Academy of Sciences, Szeged H-6726, Hungary \\ e Department of Oncotherapy, Faculty of Medicine, University of Szeged, H-6720, Hungary
}

\section{A R T I C L E I N F O}

\section{Article history:}

Received 2 April 2015

Received in revised form 8 June 2015

Accepted 13 June 2015

Available online 16 June 2015

\section{Keywords:}

Gamma-linolenic acid

Irradiation

Lipid droplet

Glioma

Gene expression

Lipidomic analysis

\begin{abstract}
A B S T R A C T
Previous studies have demonstrated that gamma-linolenic acid (GLA) is effective against glioma cells under both in vitro and in vivo conditions. In the present study we determined how GLA alone or in combination with irradiation alters the fatty acid (FA) and lipid profiles, the lipid droplet (LD) content, the lipid biosynthetic gene expression and the apoptosis of glioma cells. In GLA-treated cells direct correlations were found between the levels of various FAs and the expression of the corresponding FA biosynthetic genes. The total levels of saturated and monosaturated FAs decreased in concert with the down-regulation of FASN and SCD1 gene expression. Similarly, decreased FADS1 gene expression was paralleled by lowered arachidonic acid $(20: 4 n-6)$ and eicosapentaenoic acid (20:5n-3) contents, while the down-regulation of FADS2 expression was accompanied by a diminished docosahexaenoic acid $(22: 6 n-3)$ content. Detailed mass spectrometric analyses revealed that individual treatments gave rise to distinct lipidomic fingerprints. Following uptake, GLA was subjected to elongation, resulting in dihomo-gamma-linolenic acid (20:3n-6, DGLA), which was used for the synthesis of the LD constituent triacylglycerols and cholesteryl esters. Accordingly, an increased number of LDs were observed in response to GLA administration after irradiation. GLA increased the radioresponsiveness of U87 MG cells, as demonstrated by an increase in the number of apoptotic cells determined by FACS analysis. In conclusion, treatment with GLA increased the apoptosis of irradiated glioma cells, and GLA might therefore increase the therapeutic efficacy of irradiation in the treatment of gliomas.
\end{abstract}

(c) 2015 Elsevier B.V. All rights reserved.

\section{Introduction}

Numerous studies have demonstrated that polyunsaturated fatty acids (PUFAs) have anti-cancer activities and can be used as cancer chemopreventive agents. The application of PUFAs has been confirmed to exert beneficial effects against glioma under both in vitro and in vivo conditions [1-9]. However, the exact mechanisms underlying the anti-tumor effects of PUFAs are complex and remain poorly understood. PUFAs induce oxidative stress through the generation of reactive oxygen species (ROS) which results in enhanced lipid peroxidation

\footnotetext{
* Corresponding author at: Laboratory for Functional Genomics, Institute of Genetics, Biological Research Center of the Hungarian Academy of Sciences, Szeged H-6726, Hungary.

E-mail address: puskas.szbk@gmail.com (L.G. Puskás).

1 Contributed equally.

2 Co-senior authors.

${ }^{3}$ www.avidinbiotech.com.
}

and decreased cell survival. This may be one of the main indirect causes of the cytotoxicity of PUFAs [10]. Additionally, ROS can induce a dysfunction of the mitochondria, which can lead to apoptosis [11]. Interestingly, PUFA treatment can enhance the radiosensitivity of cancerous cells, while it has proved to be radioprotective to normal cells [12,13]. This phenomenon has been attributed to the possible differences in sensitivity of cancer and normal cells in combating lipid peroxidation products [13].

It was shown earlier that cyclooxygenase inhibitors diminish the gamma-linolenic acid (GLA)-induced radiosensitivity of astrocytoma cells, and PUFAs may therefore induce radioresponsiveness through prostanoid synthesis, though their cytotoxicity may not be related to this effect [9]. There have been efforts to find radiosensitizers to intensify radiotherapy for glioma, but none have resulted in improved survival [14].

PUFA availability is known to alter the composition of the membranes, their fluidity and the way microdomains organize [15]. This causes the release of heat shock signals, which lead to heat shock protein (HSP) expression [15]. Membrane modulations, signal 
pathways from membranes to hsp genes and the HSPs themselves play essential roles in the etiology of several diseases, including cancer [16].

Of the various PUFAs, the most extensive studies have been performed with GLA in glioma treatment [1-3]. GLA enhances the effects of radiotherapy on cancerous cells, at the same time exerting a cytoprotective effect on normal cells [2,3]. In clinical experiments, the infusion of GLA increased the length of survival [3]. Our previous studies of the mechanisms by which GLA and other PUFAs in combination with irradiation exert their effects on glioma cells revealed the additive effect of GLA treatment and irradiation on cell proliferation inhibition, as confirmed by the lactate dehydrogenase activity and gene expression profiling [4].

The balance between fatty acid (FA) alimentation, synthesis, storage and apoptosis influences cancer development. In comparison with normal cells, de novo FA synthesis is activated during carcinogenesis $[17,18]$. In consequence of their continuous proliferation, cancer cells need increased FA biosynthesis to support lipoprotein synthesis and cell membrane biogenesis [18,19]. Chajès et al. [17] found that FA synthesis for cell proliferation is dispensable when FAs are available in the medium. Furthermore, in normal cells, FA synthesis is inhibited by exogenous FAs, while in cancerous cells the synthesis is intensely active [19]. Although the correlation between the lipid metabolism and cancer development has not yet been deciphered, alterations in the lipid metabolism and the accumulation of lipids in lipid droplets (LDs) have been implicated in carcinogenesis [13]. LD accumulation occurs in prenecrotic cancer tissues in vivo, and LDs can therefore, serve as in vivo markers of cancer [20]. Moreover, small-molecule drugs that target the LDs proved to induce endoplasmic reticulum and oxidative stress and to exert cytotoxic effects in diverse cancer cell lines [21], and to suppress hepatocellular carcinoma in vivo [22].

The possible application of GLA as an adjuvant in clinical experiments on the treatment of glioma necessitates a better understanding of the overall effects of co-exposure to irradiation and GLA. In this study we set out to investigate the dynamics of the FA and lipid metabolism and storage and the rate of apoptosis by performing experiments on the U87 MG glioblastoma cell line exposed to irradiation and/or GLA. FA and lipid profiles were recorded by means of mass spectrometric techniques and the expression of several genes present in the biosynthetic pathway of FAs was evaluated, including genes coding enzymes responsible for de novo synthesis of saturated and monounsaturated FAs (FASN, SCD1 and SCD5) and desaturases and elongases that participate in the synthesis of PUFAs (FADS1, FADS2, ELOVL1, ELOVL2 and ELOVL5). We additionally measured the expression of several heat shock genes, monitored the LD content by fluorescence microscopy, and determined the rate of apoptosis by FACS analysis.

\section{Results}

\subsection{Effects of GLA treatment and irradiation on the rate of apoptosis}

To confirm the apoptosis-inducing effects of GLA and irradiation, FACS analysis was performed. As shown in Fig. 1, dose-dependent increases in apoptotic cells occurred in response to $5 \mathrm{~Gy}$ or $10 \mathrm{~Gy}$ of irradiation. Similarly, GLA alone significantly increased the number of apoptotic cells and, in parallel, decreased the total number of living cells. When GLA was used in combination with irradiation, moderate increases in apoptotic cell number were registered. As expected, the most pronounced effects on cell survival and apoptosis were seen in samples treated with $10 \mathrm{~Gy}$ irradiation and GLA.

\subsection{Total FA analysis of GLA-treated cells by gas chromatography-mass spectrometry}

To determine how GLA treatment influences the total FA profile of U87 MG glioma cells, we used GC-MS (Fig. 2). GLA-treated cells exhibited decreases in the total saturated and monounsaturated FAs (SFAs and MUFAs, respectively; Fig. 2, inset). As expected, the level of GLA increased on GLA treatment. The analysis also revealed a very significant, over 7-fold increase in the amount of the elongated derivative dihomo-gamma-linolenic acid (20:3n-6; DGLA). In parallel, the contents of arachidonic acid (AA), eicosapentaenoic acid (EPA) and docosahexaenoic acid (DHA) decreased.

\subsection{Effects of irradiation, GLA and their combination on the lipid molecular species profile}

To reveal the changes elicited by irradiation and/or GLA treatment in the lipidomes of U87 MG cells, high-sensitivity, high-resolution shotgun profiling was carried out on an Orbitrap Elite hybrid tandem mass spectrometer. Altogether almost 250 lipid molecular species were identified and quantified, each of which accounted for more than $0.4 \%$ within its lipid class (Supplementary Table 1S). The data generated (i.e. 248 molecular species $\times 2 \times 3$ different treatments $\times 3$ independent replicates) served as a basis for partial least squares discriminant analysis (PLS-DA) to test for possible lipid differences caused by the various treatment conditions and also to assess overall experimental variation. PLS-DA is a supervised multivariate classification method that is useful for reducing multidimensional data to lower dimensions, thereby simplifying the visualization of complex datasets for exploratory analysis. It was confirmed by cross-validation and permutation test that the achieved good separation (Fig. 3 ) is based on real signals. The PLS-DA analysis indicated that the two highest ranking components, components 1 and 2 , accounted for $58 \%$ and $9 \%$, of the total variance, respectively. It clearly emerged that component 1 captured most of the variations
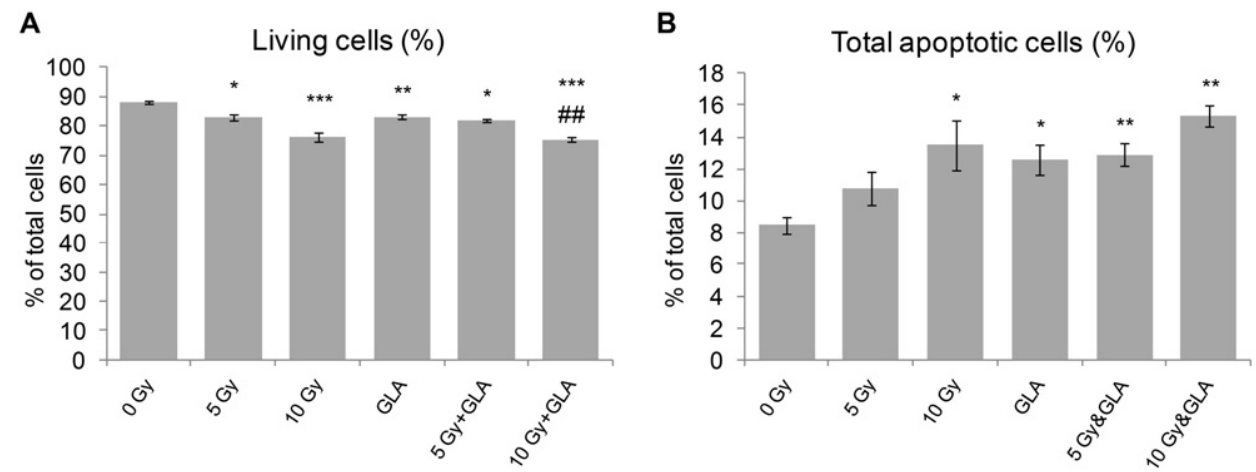

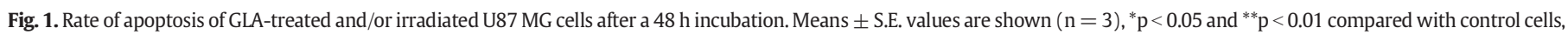
$\# \#$ p $<0.01$ compared with GLA-treated cells. 


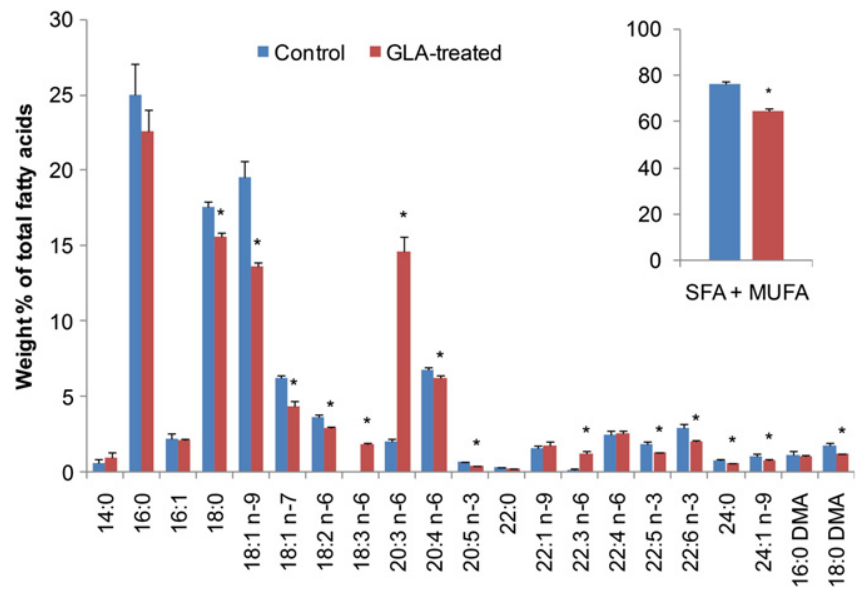

Fig. 2. Total FA analysis of control and GLA-treated U87 MG cells by GC-MS. Data are expressed as weight $\%(\mathrm{~m} / \mathrm{m})$ of total FA and expressed as means \pm S.D. $(n=3)$, ${ }^{*} \mathrm{p}<0.025$ (FDR $<0.05$ calculated according to Benjamini and Hochberg [82]). DMA denotes dimethyl acetal from plasmalogen species. Inset: Sum of total saturated (SFA) and monounsaturated FAs (MUFA).

between the GLA-non-treated and GLA-treated samples, whereas component 2 dose-dependently separated the gamma-ray treatments. PLS-DA therefore demonstrated that the individual treatments gave rise to distinct lipidomic fingerprints.

The observed changes were further analyzed by comparing the molecular species patterns of the individual treatments, which demonstrated 183 statistically-significant distinctions. The heatmap of selected species (significant in ANOVA, p $<0.007$, FDR $<0.01$, Fig. 4) convincingly illustrates that the GLA supplementation dramatically rearranged the lipidome of U87 cells.

In order to select appropriate alterations of explanatory relevance, data were grouped according to changes in lipid class, fatty acyl chain length and/or degree of unsaturation. As expected, GLA treatment alone considerably increased the relative amounts of phospholipid species containing 3 or 4 double bonds (Fig. 5). In agreement with the GCMS analysis, electrospray ionization-MS/MS fragmentation experiments revealed that, following the uptake, GLA is subjected to elongation, and

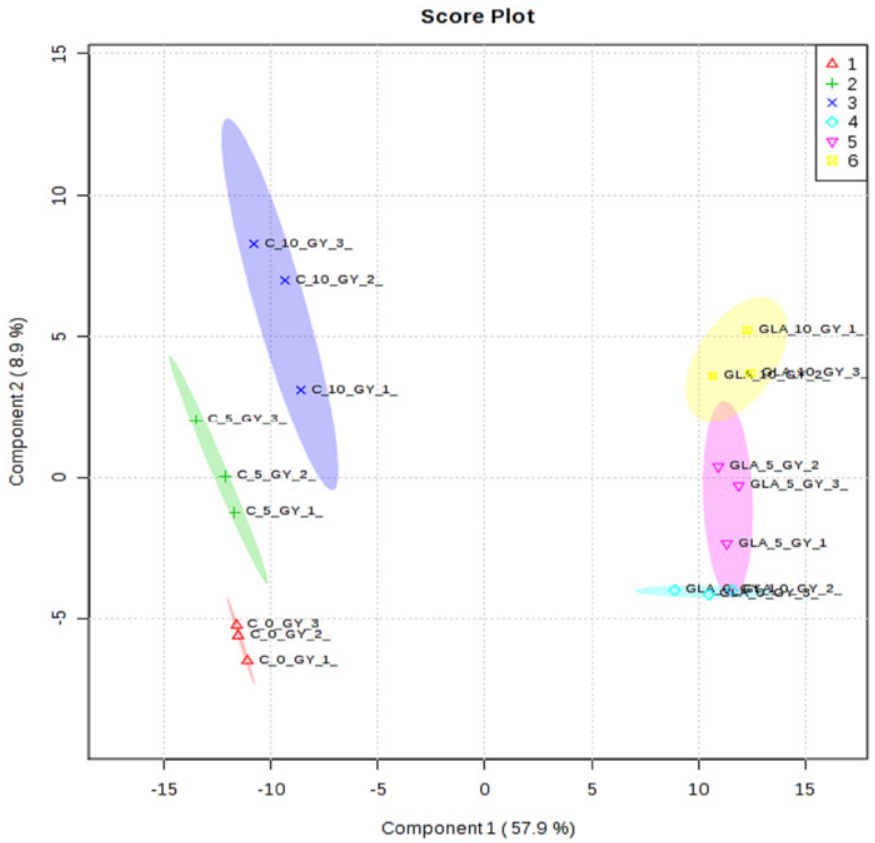

Fig. 3. Partial least squares discriminant analysis (PLS-DA) score plots of lipidomic datasets. Values from 3 independent experiments are shown for control and treated cells.

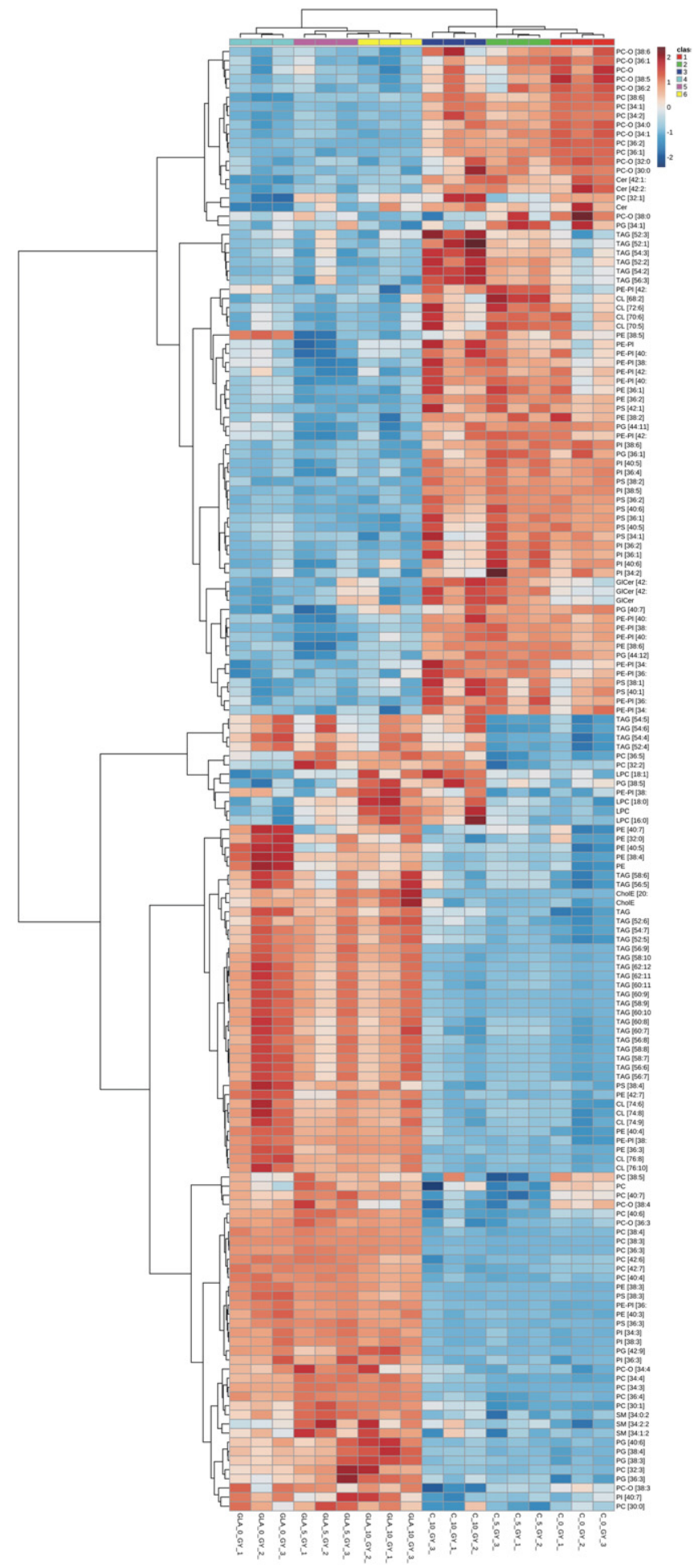

Fig. 4. Heatmap representation of selected lipid molecular species of irradiated and/or GLA-treated U87 MG cells. Selection was based on significance in ANOVA, $p<0.007$, $\mathrm{FDR}<0.01$.

the main FA incorporated was therefore DGLA (Figs. 2 and 5 inset). Interestingly, no further desaturation (i.e. increase in AA) was observed. A significant fraction of GLA, after elongation to DGLA, was redirected to lipid droplets and incorporated into triacylglycerol species (TAGs) (Fig. 6A) and cholesteryl ester species (CholEs) (Fig. 6B).

Lipid class analysis of control and irradiated cells in combination with or without GLA treatment did not reveal substantial changes in the amounts of major membrane lipids (Fig. 7). Irrespectively of 


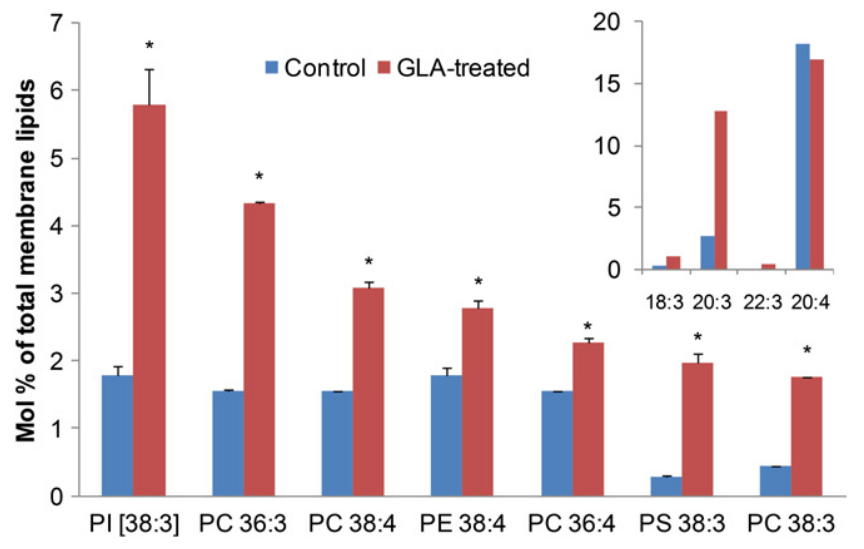

Fig. 5. Changes in the relative amount of phospholipid species containing 3 or 4 double bonds in response to GLA treatment of U87 MG glioma cells. Data are expressed as mol\% of total polar lipids and are presented as means \pm S.D. $(n=3),{ }^{*} \mathrm{p}<0.012$ (FDR $<0.01 \mathrm{cal}-$ culated according to Storey and Tibshirani [81]). Inset: representative FA profile resulting from ESI-MS/MS fragmentation of phospholipids. PC, phosphatidylcholine; PI, phosphatidylinositol; PS, phosphatidylserine.

GLA treatment, the only noteworthy common lipid alteration due to irradiation was the dose-dependent increase in the content of lysophosphatidylcholine (LPC) (Fig. 7). At a lipid species level, each molecular species of LPC (Fig. 8) displayed a gradual accumulation in response to gamma-ray treatment both in control and in GLA-treated samples.

In order to identify lipidomic changes that may distinguish the effects of irradiation + GLA treatment from those obtained with irradiation alone, clustering, heatmap and ANOVA were run separately on the corresponding datasets. The heatmap representations of selected species, based on ANOVA results, revealed essentially different patterns of lipid molecular species for the non-treated and GLA-supplemented cells (Fig. 9). In the GLA-treated samples, the irradiation resulted in an enhanced loss of several species of phosphatidylethanolamine (PE) and phosphatidylserine (PS). Specific changes in the molecular species of these phospholipid classes are visualized by grouping these species according to the total number of their double bonds (Fig. 10). As concerns the alterations in storage lipids, irradiation alone induced a dose-dependent accumulation of TAG species and this elevation was higher in species esterified with PUFAs (Fig. 11). GLA-co-treated cells displayed elevated levels of TAG species containing PUFAs (double bond number 4-12) in comparison with non-treated ones. In contrast with non-supplemented cells, irradiation + GLA supplementation did not induce further elevation of the TAG pools.

\subsection{Evaluation of lipid droplet content of treated cells}

We used a live cell LD-specific dye, AC-202, to evaluate the average cellular LD content of GLA- and/or irradiation-treated U87 MG cells, and control, untreated cells. Microscopic analysis revealed significant increases in LD amounts in GLA-treated and irradiated cells as compared with irradiated control samples (Fig. 12A). Irradiation decreased the LD content of the cells, while in GLA-treated cells, irradiation dosedependently increased the LD amount. Representative LD images of irradiated cells with or without GLA treatment are presented in Fig. 12.

\subsection{Gene expression analysis}

In order to determine the effects of GLA and/or irradiation on FA biosynthetic genes, the expression of FASN, ELOVL1, ELOVL2 and ELOVL5 genes (Fig. 13), and SCD1, SCD5, FADS1, FADS2 and FADS3 genes (Fig. 14) was determined by using QRT-PCR.

While irradiation with 5 Gy demonstrated only a tendency to enhance the FA synthase FASN expression, the higher dose (10 Gy) up-regulated its expression significantly (Fig. 13A). In contrast, GLA treatment decreased the expression of FASN significantly. Co-exposure of U87 cells to $10 \mathrm{~Gy}+$ GLA caused a significantly higher expression level than that in GLA-treated cells (Fig. 13A). Irradiation + GLA treatment did not significantly alter the expression of FA elongases: ELOVL1, ELOVL2 and ELOVL5 (Fig. 13B, C, D). Only ELOVL5 was expressed at a significantly higher level in samples exposed to irradiation + GLA as compared with samples treated with GLA alone (Fig. 13D).

Desaturases SCD1 and SCD5 were significantly down-regulated $(\mathrm{p}<0.01)$ after GLA treatment (Fig. 14A, B), whereas their expression

\section{A}

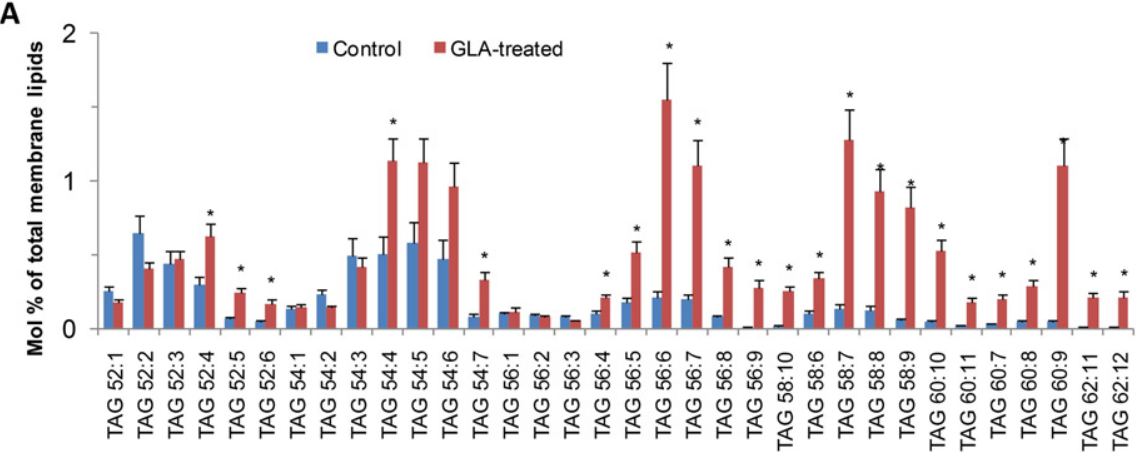

B

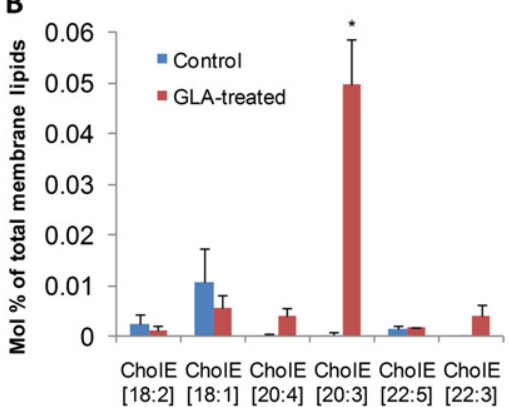

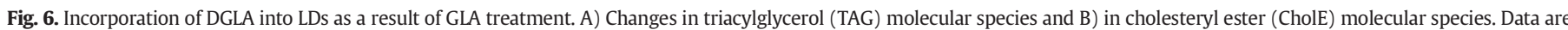
expressed as mol\% of total polar lipids and are presented as means \pm S.D. $(\mathrm{n}=3),{ }^{*} \mathrm{p}<0.012$ (FDR $<0.01$ calculated according to Storey and Tibshirani [81]). 


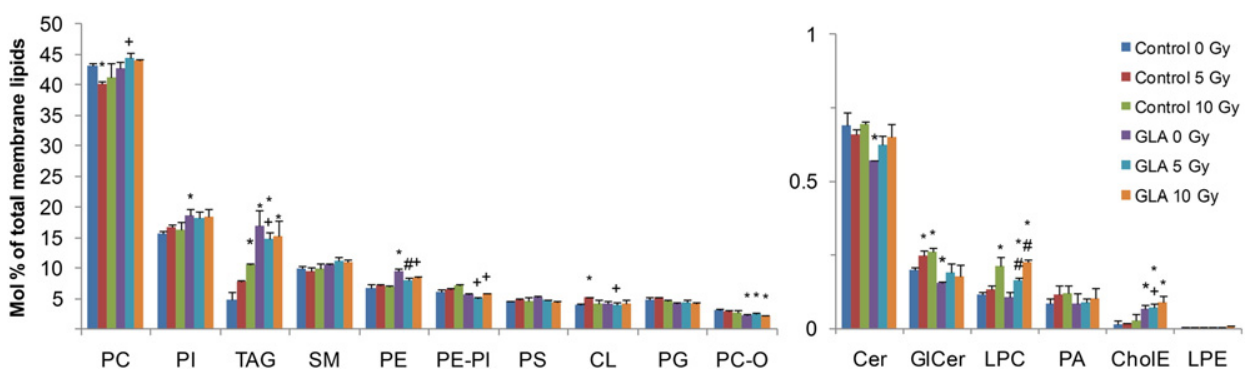

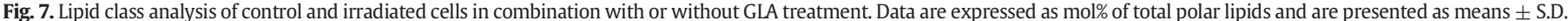

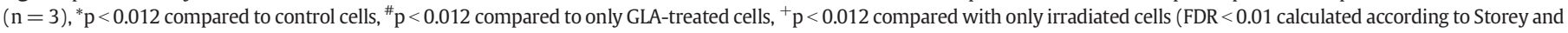

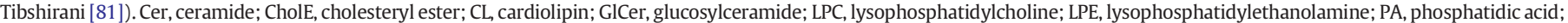

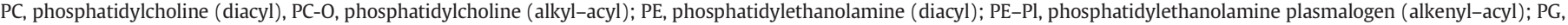
phosphatidylglycerol; PI, phosphatidylinositol; PS, phosphatidylserine; SM, sphingomyelin; TAG, triacylglycerol.

was not significantly altered when irradiation was applied together with GLA.

GLA treatment alone also diminished the expression of desaturases FADS1 and FADS2 ( $<0.05)$ (Fig. 14C, D). Similarly as for SCD1 and $S C D 5$, the GLA-induced down-regulation was reversed to the control levels in cells co-treated with GLA + irradiation (Fig. 14C, D). Similar trends were observed in case of FADS3, although these changes were not statistically significant (Fig. 14E).

Since our results indicated that LD formation was modulated by the applied treatments, we monitored the expression of PLIN3, the perilipin-3 gene which has a special role in LD formation and stability. The PLIN3 expression was increased significantly in irradiated cells ( $p<0.01$ ) (Fig. 15A), whereas GLA treatment was ineffective. Co-treatment decreased the radiation-induced over-expression of PLIN3 (Fig. 15A).

Several heat shock protein coding genes were also analyzed. GLA treatment proved to induce HSP9OAA1 expression by more than 3-fold (Fig. 15B), but irradiation or irradiation + GLA had no effect on its expression. While the expression of HSPB1 was moderately increased after the treatments, HSPB2 was unaffected (Fig. 15C, D). The statistically not significant increase in HSPB2 expression in response to irradiation was normalized by GLA, while the elevated HSPB1 level induced by irradiation remained the same after GLA treatment.

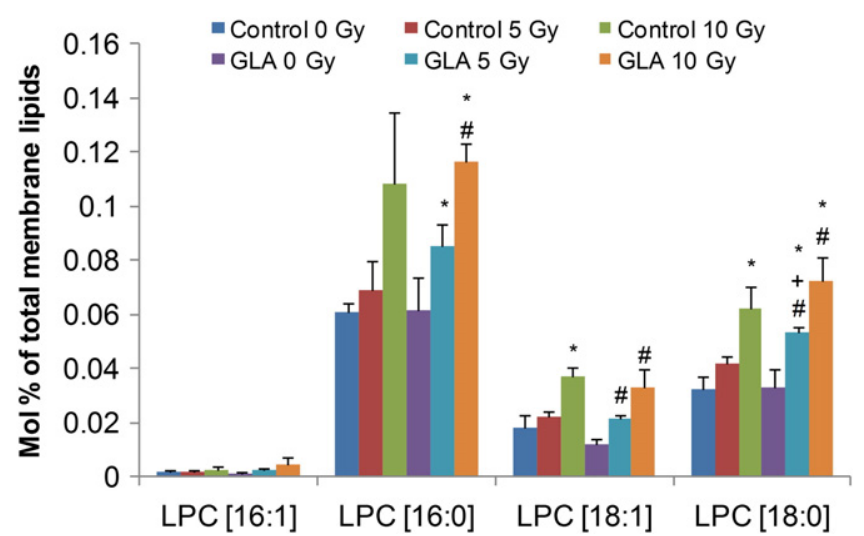

Fig. 8. Changes in lipid molecular species of lysophosphatidylcholine (LPC) in response to irradiation with or without GLA treatment. Data are expressed as mol\% of total polar lipids and presented as means \pm S.D. $(n=3),{ }^{*} \mathrm{p}<0.012$ compared with control cells, ${ }^{*} \mathrm{p}<0.012$ compared with only GLA-treated cells, ${ }^{+} \mathrm{p}<0.012$ compared to only irradiated cells (FDR $<0.01$ calculated according to Storey and Tibshirani [81]).

\section{Discussion}

The results of earlier cellular assays and in vivo experiments led to the conclusion, that PUFAs could be useful adjuncts to irradiation in cancer treatment $[3,4,12,23]$. We found that both GLA treatment and irradiation induced apoptosis induction in U87 MG glioma cells, even after incubation for $48 \mathrm{~h}$. The moderate rate of apoptosis detected may be due to the relatively short incubation time ( $48 \mathrm{~h}$ ). However, our results correlate with the previous finding that a longer period is needed for apoptosis induction. In a human ADF astrocytoma cell line, for example, fractionated irradiation (5 Gy on 4 successive days) caused apoptosis, while exposure to $12.5 \mathrm{~Gy}$ caused necrosis [24].

In order to detect early changes in gene expression and in FA and lipid profiles we analyzed all of our samples $48 \mathrm{~h}$ post-treatment. Total FA analysis revealed that the levels of SFAs and MUFAs decreased in concert with the down-regulation of the FASN and SCD1 genes following GLA treatment. FASN is a cytoplasmic multifunctional complex with various enzymatic activities [25]. It is the only human enzyme which can synthesize FAs de novo [26]. It is over-expressed or hyperactive in numerous types of cancer, and its expression level correlates with tumor development, aggressiveness and the rate of metastasis [32]. While FASN inhibition does not influence the growth and development of normal cells, it arrests the cell cycle and induces programmed cell death in cancer cells [29] and reduces tumor cell growth in vivo [27, 28]. FASN is considered to be an oncogene and, due to its suggested role in cancer pathogenesis, it is also a potential therapeutic target [30, 31]. We found that irradiation caused an up-regulation, while GLA treatment resulted in a down-regulation of FASN (Fig. 13A). Although FASN expression in tumors seemed to be unresponsive to nutritional signals [27], in the present study irradiation-induced FASN over-expression was attenuated by GLA treatment.

SCD1 is a $\triangle 9$ desaturase that catalyzes the synthesis of MUFAs from SFAs [32]. SCD1 regulates the speed of lipogenesis and cell proliferation, influences the membrane fluidity by altering the SFA:MUFA ratio, and affects membrane lipid domain compositions, thereby changing the activities of plasma membrane proteins $[33,34]$. It has been suggested that SCD1 may have an anti-apoptotic effect, while its inhibition causes apoptosis [19,35]. SCD5 enhances the production of CholE and PC, and diminishes PE and TAG synthesis [33]. Sinner et al. [33] propose SCD5 as a therapeutic target in the treatment of brain tumors. In breast cancer patients with a pathological complete response the SCD5 expression was observed to be decreased significantly [36]. In our study GLA decreased the expressions of SCD1 and SCD5 more than 4-fold, while irradiation was ineffective. Interestingly, irradiation attenuated the effect of co-applied GLA (Fig. 14A, B).

Previous studies have investigated the lipid composition following GLA supplementation. In rats, no changes were observed in the GLA or DGLA content of tumors [37]. In vitro studies reported on changes in 

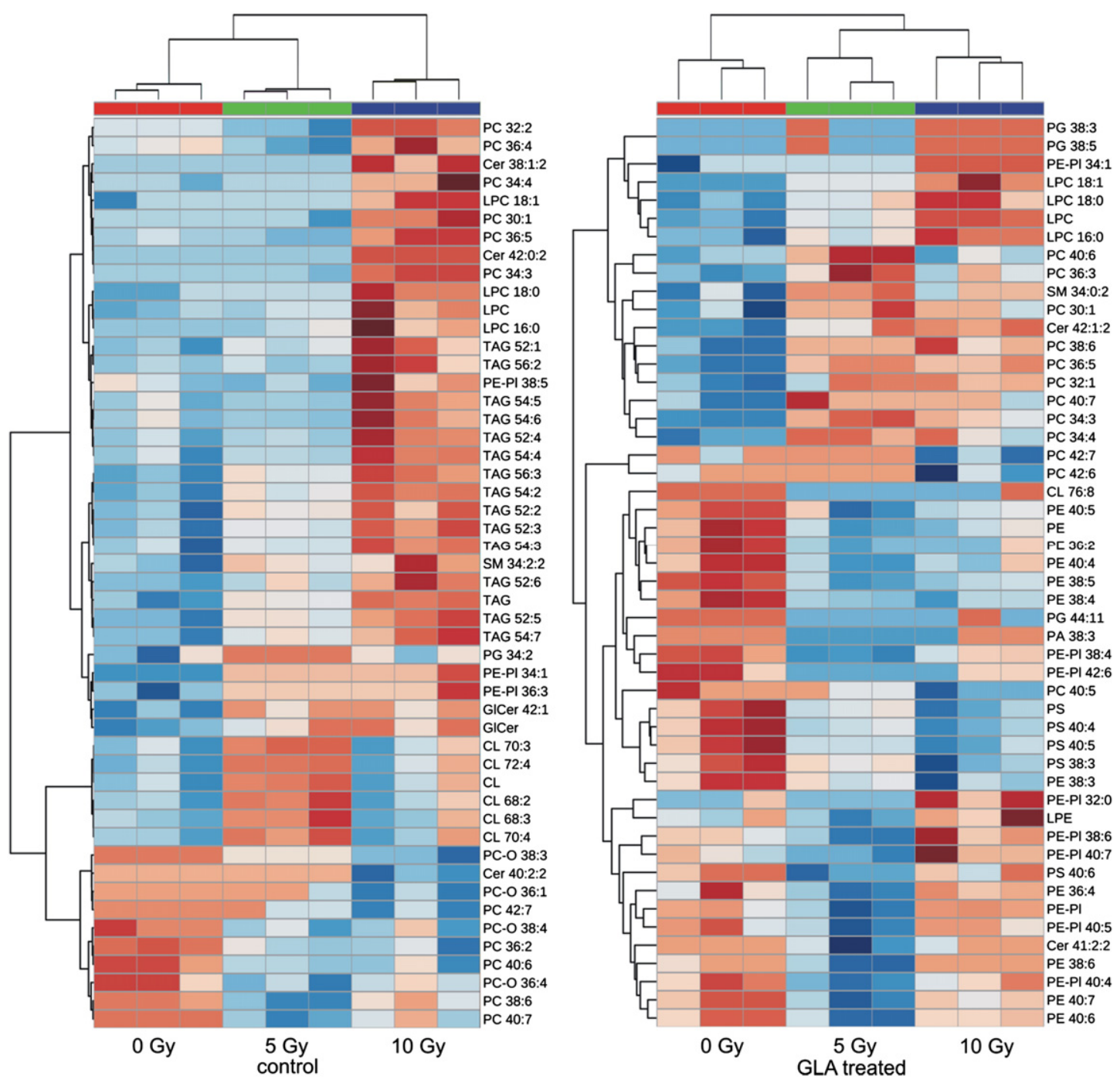

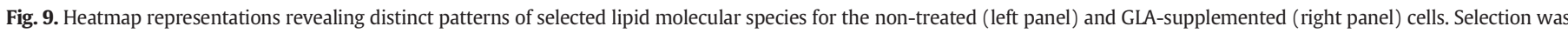
based on the significance in ANOVA, $\mathrm{p}<0.007$, FDR $<0.01$.

lipid content of 36B10 astrocytoma [10] and C6 glioma cells after treatment with GLA [38]. Following incorporation in C6 glioma cells, GLA was elongated and desaturated and effectively replaced MUFAs, while the SFA concentration remained constant [38]. In the present study the GLA content of U87 MG cells was markedly increased after GLA treatment (from $0 \%$ to ca. $2 \%$ of the total FAs). Most of the GLA was then subjected to elongation to DGLA and incorporated effectively in this form as a result of GLA treatment (the total FA\% of DGLA increased from $2 \%$ to $15 \%$ ), though the corresponding elongase (ELOVL5) expression remained unchanged after incubation for $48 \mathrm{~h}$. In contrast with other studies on neuroblastoma [39], astrocytoma [9] and glioma [3, 38] models, where DGLA was found to be further desaturated to AA, in our experiments the amount of AA was even lowered, this correlating with the down-regulation of FADS1. Moreover, diminished levels of EPA and DHA may be related to FADS1 and FADS2 down-regulation, respectively. These findings are in accordance with the observation
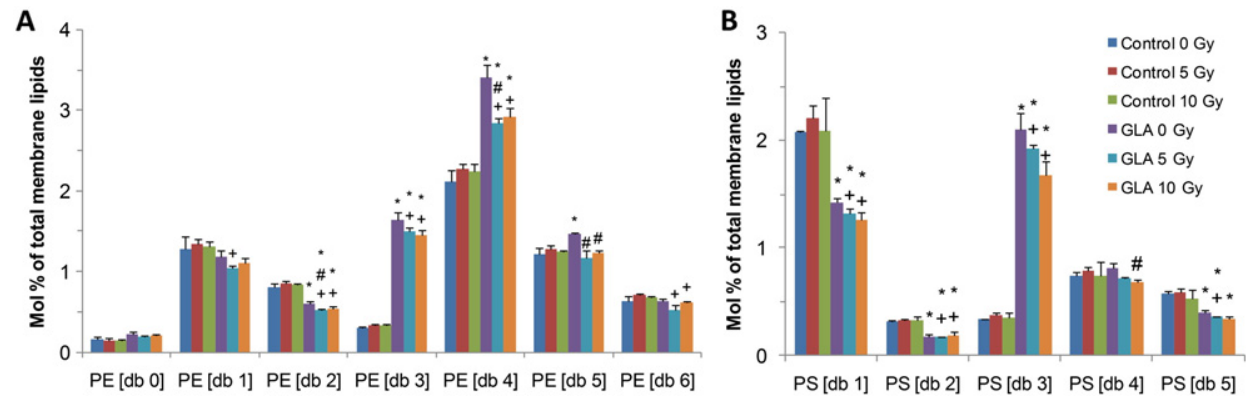

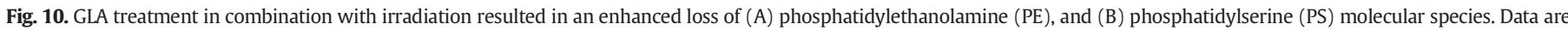

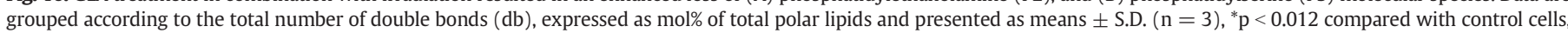
${ }^{\#} \mathrm{p}<0.012$ compared with only GLA-treated cells, ${ }^{+} \mathrm{p}<0.012$ compared with only irradiated cells (FDR $<0.01$ calculated according to Storey and Tibshirani [81]). 


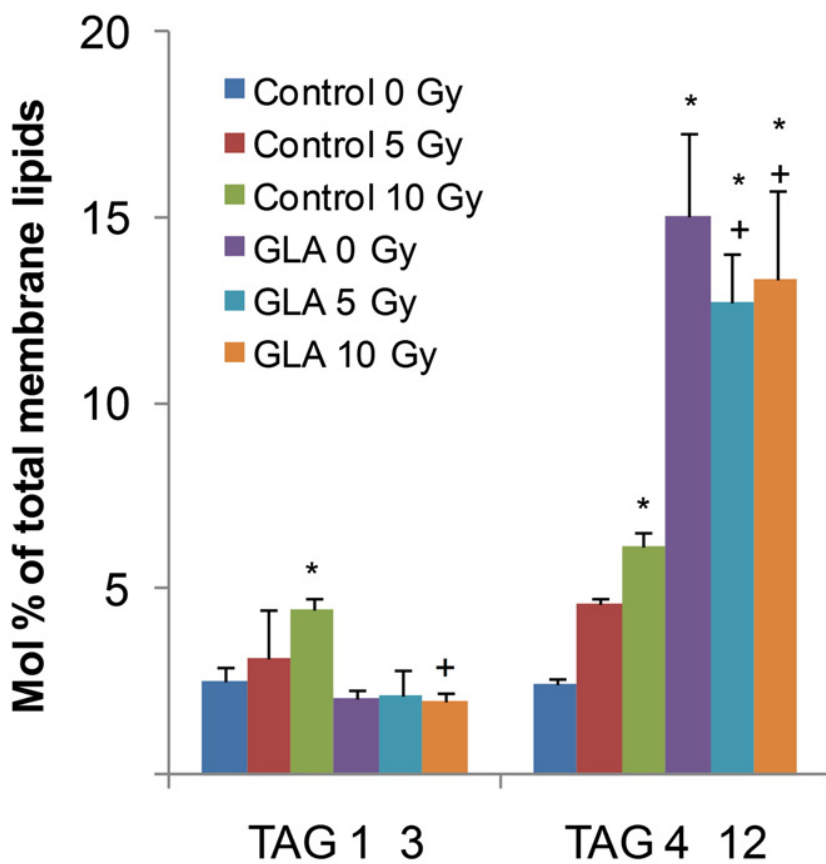

Fig. 11. Alterations in triacylglycerol (TAG) species in GLA- and/or irradiation-treated cells. Data are expressed as mol\% of total polar lipids and are presented as means \pm S.D. $(n=3)$, ${ }^{*} \mathrm{p}<0.012$ compared with control cells, ${ }^{*} \mathrm{p}<0.012$ compared with only GLA-treated cells, ${ }^{+} \mathrm{p}<0.012$ compared with only irradiated cells (FDR $<0.01$ calculated according to Storey and Tibshirani [81]). that dietary $n-6$ and $n-3$ PUFAs suppress the transcription of several glycolytic and lipogenic enzyme-coding genes [40]. The suppressed expression of lipogenic genes may be connected to a decrease in the nuclear form of the transcription factor SREBP-1 (sterol-regulatory-element-binding protein 1), the master regulator of lipogenic genes [41].

FADS1 ( $\triangle 5$ desaturase) and FADS2 ( $\triangle 6$ and $\Delta 8$ desaturase) are indispensable for PUFA synthesis [32,42]. In agreement with previous findings, both were down-regulated after GLA treatment in our experiments (Fig. 14D, E). Several studies have reported that FADS2 was down-regulated following PUFA treatment both in vitro and in vivo [43-48]. In contrast with FADS1 and FADS2, the expression of FADS3, which has been implicated in the maintenance of lipid homeostasis $[49,50]$, was not affected by GLA treatment.

Elongases are specific for substrates with a given chain length and saturation [51]. ELOVL1, ELOVL2 and ELOVL5 have different substrate specificities [25,52]. ELOVL2 elongates C20 and C22 PUFAs, ELOVL5 C18-C20 PUFAs, and ELOVL1 C18:0-C26:0 FAs [25,52-54]. The expression of the examined ELOVL1, ELOVL2 and ELOVL5 was not altered significantly by any of the applied treatments (Fig. 13D).

PUFA treatment may influence the membrane fluidity by altering the saturated:unsaturated FA ratio and in mammalian cells a correlation has been reported between membrane perturbations and heat shock response [55]. We therefore followed the changes in expression of several hsp genes. The applied treatments did not alter the expression of HSPB2 significantly, whereas irradiation (10 Gy), GLA treatment and their combinations caused a significant up-regulation of HSPB1. Similarly to our results, Bucci et al. observed the dose-dependent up-regulation of HSPB1 in the ADF human astrocytoma cell line; 5 Gy irradiation did not affect its expression, whereas it was up-regulated in $12.5 \mathrm{~Gy}$-irradiated cells [24]. Hsp90 is a therapeutic target in glioblastoma multiforme: its
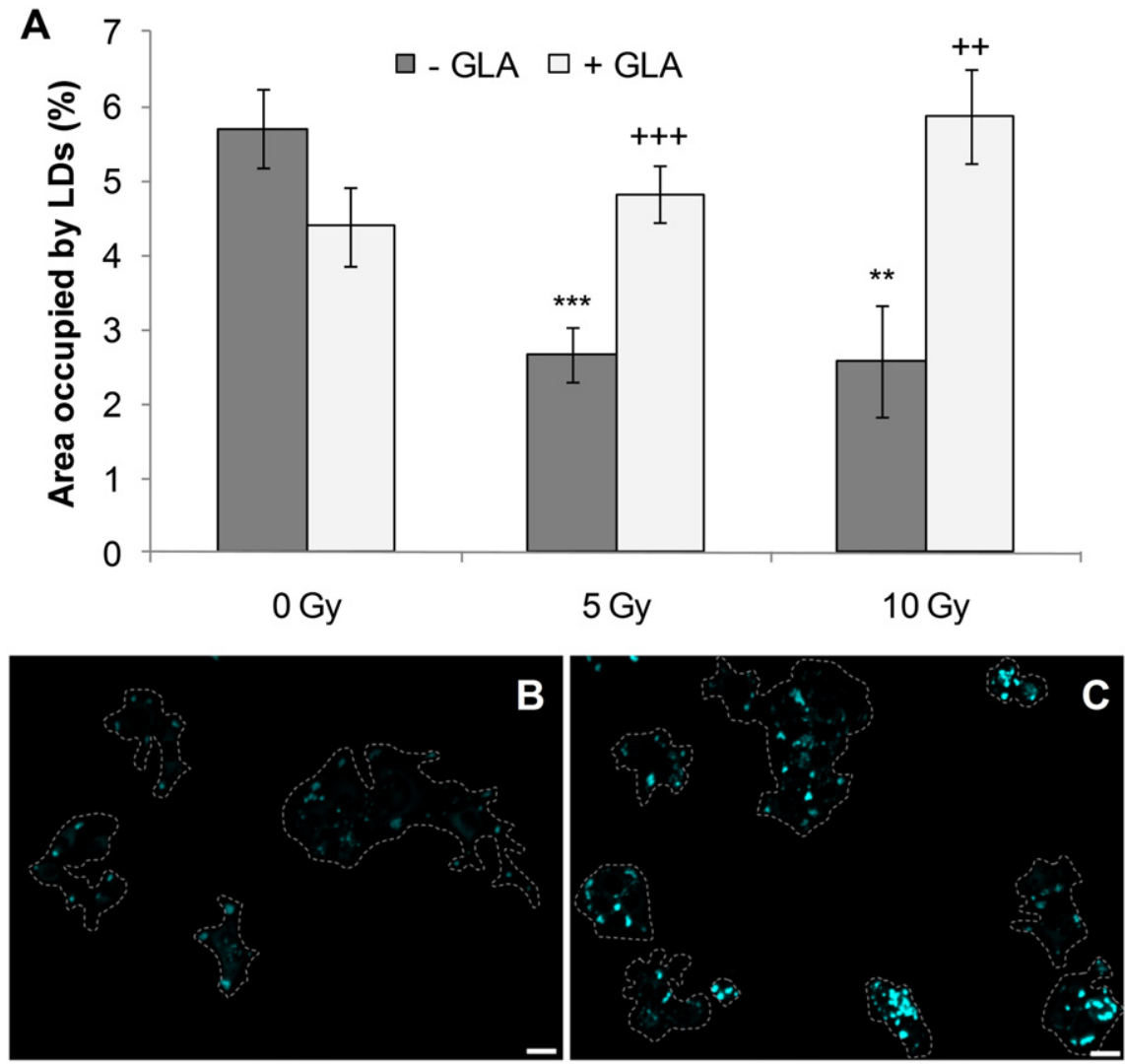

$10 \mathrm{~Gy}$

$10 \mathrm{~Gy}+\mathrm{GLA}$

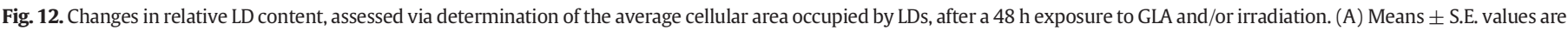

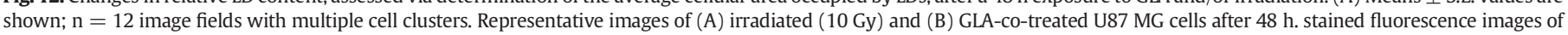
(B) 10 Gy-treated control and (C) GLA-co-treated U87 MG cells after $48 \mathrm{~h}$. Dashed lines indicate cell cluster borders drawn with the aid of transmitted light images. Scale bar: $10 \mu$ m. 

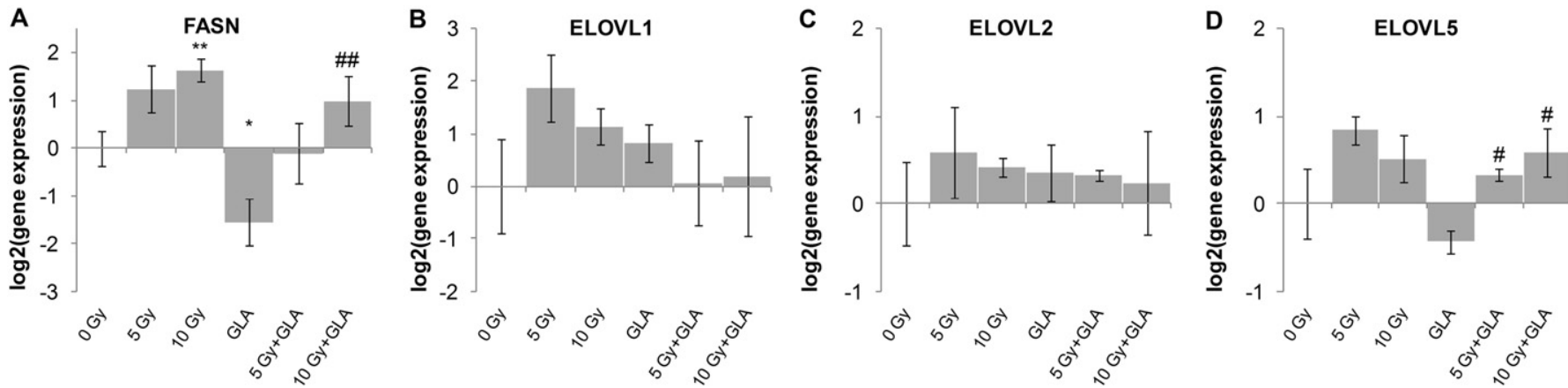

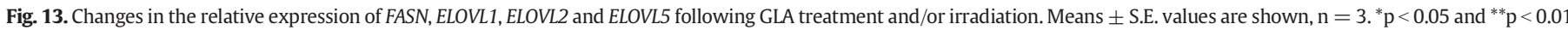
compared with control cells, ${ }^{\#} \mathrm{p}<0.05$ and ${ }^{\# \#} \mathrm{p}<0.01$ compared with only GLA-treated cells.

inhibition may "repair" several signaling pathways and could affect more than a hundred targets which regulate the cell cycle, proliferation and apoptosis [56]. We found that GLA treatment alone affected the expression of HSP90AA1 while irradiation + GLA normalized its expression level to the baseline.

To recognize lipid changes as a consequence of irradiation and/or GLA treatment, lipidomic datasets were analyzed by using the data-mining PLS-DA method; this resulted in good separation of the experiments into six distinguishable clusters, according to the six different treatment combinations (Fig. 3). The first component accounted for almost $60 \%$ of the total variance. It clearly separated the samples treated with and without GLA. Indeed, GLA treatment was able to reshape the whole lipidome essentially, as a result of its incorporation (mainly in the form of DGLA) into both the membrane and the storage lipids, i.e. increasing the relative levels of PUFA-containing phospholipids and also TAGs and CholEs, with parallel decreases in MUFA- and SFA-containing species (Fig. 4 and Supplementary Table 1S).

It is well established that ionizing radiation strongly induces the generation of ROS, and lipids are one of the major targets for these radicals [57]. The PUFA-containing lipids are mostly affected by the initial formation of phospholipid hydroperoxides and secondary lipid peroxidation products, such as thiobarbituric acid-reactive substances and 4-hydroxynonenal [58]. It has been proposed that phospholipase $\mathrm{A}_{2}\left(\mathrm{PLA}_{2}\right)$ is activated by oxidized phospholipids, and protects membranes from damage by peroxidation by preferentially cleaving the oxidatively modified species $[59,60]$. Indeed, PLA $_{2}$ is known to be activated by irradiation in order to repair the attacked phospholipids and generate lipid mediators contributing to survival. PLA $\mathrm{PL}_{2}$ is therefore able to diminish the efficacy of radiotherapy in tumor models [61]. On the other hand, PS is extremely sensitive to peroxidation, and the oxidation of PS seems to be important for its externalization during apoptosis and for the recognition of apoptotic cells by phagocytes [62]. We demonstrated that the irradiation of GLA-treated glioma cells resulted in an enhanced degradation of PUFA-containing species of PS and PE, with the concomitant (GLA-independent) generation of LPC. These changes together suggest an increased level of lipid oxidation, consequent $\mathrm{PLA}_{2}$ activation and the induction of the synthesis of HSPs [55, 63-65]. However, when it is taken into account that GLA treatment promoted the radiation-induced apoptosis of U87 cells (measured by Annexin $\mathrm{V}$ binding), it may be concluded that the PS externalizationmediated apoptotic processes in GLA and irradiation co-treated samples override the radioresistance and survival elicited by $\mathrm{PLA}_{2}$ activation and HSP induction. When cells cannot find sufficient resources to restore their functions, the stress may result in the initiation of apoptotic pathways and programmed cell death [55]. Interestingly, we did not detect an increase in ceramide (Cer) generation (Supplementary Table 1S) implying that the acid sphingomyelinase (ASMase)/Cer pathway is not activated. It was also reported that, this pathway is not activated in tissues exposed to fractionated (low-dose) radiotherapy [66,67]. Our findings corroborate the hypothesis of the existence of an alternative (low-dose) radiation-induced signaling pathway, involving plasma membrane-initiated signals, such as scramblase activation and PS externalization.

A significant proportion of GLA/DGLA was redirected to LDs and incorporated into TAGs (Fig. 6A) and CholEs (Fig. 6B). Very importantly, the gamma-ray treatment of control glioma cells induced a dosedependent accumulation of TAGs, and especially species esterified with PUFAs (Fig. 11). Interestingly, the GLA-treated cells per se exhibited elevated levels of TAG species containing PUFAs $(\mathrm{db} \geq 4)$. The enhanced $\mathrm{PLA}_{2}$ activation elicited by gamma-irradiation can produce "intact" (not-oxidized) PUFAs, which may be stored in the LDs, thereby serving as protective reservoirs during stress.

The biogenesis, morphology, development, trafficking of LDs, lipid storage and lipolysis are known to be determined by LD-associated proteins $[68,69]$. Since we observed significant dose-dependent increases in TAG and CholE content in response to irradiation and GLA treatment, we analyzed the possible changes in the expression of a lipid binding protein, PLIN3. Perilipins are a multi-protein family that target LD surfaces and regulate lipid storage and hydrolysis [70]. In good accord with the increased TAG content of treated cells, we found that irradiation
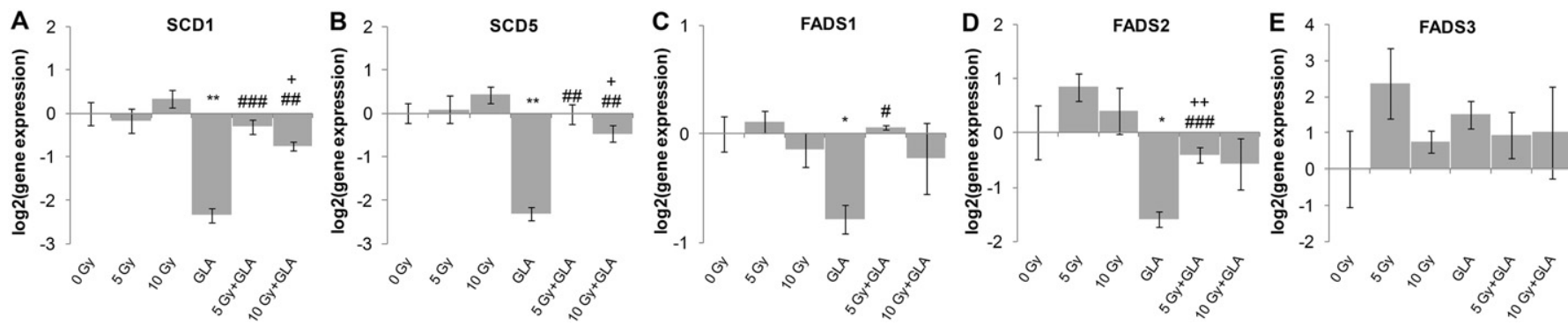

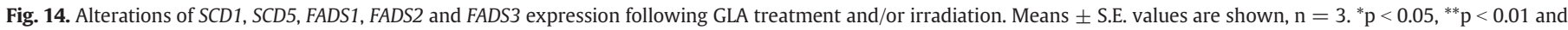

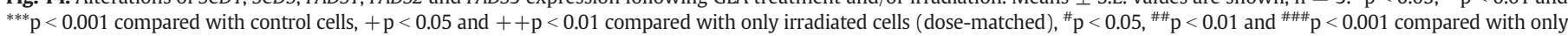
GLA-treated cells. 

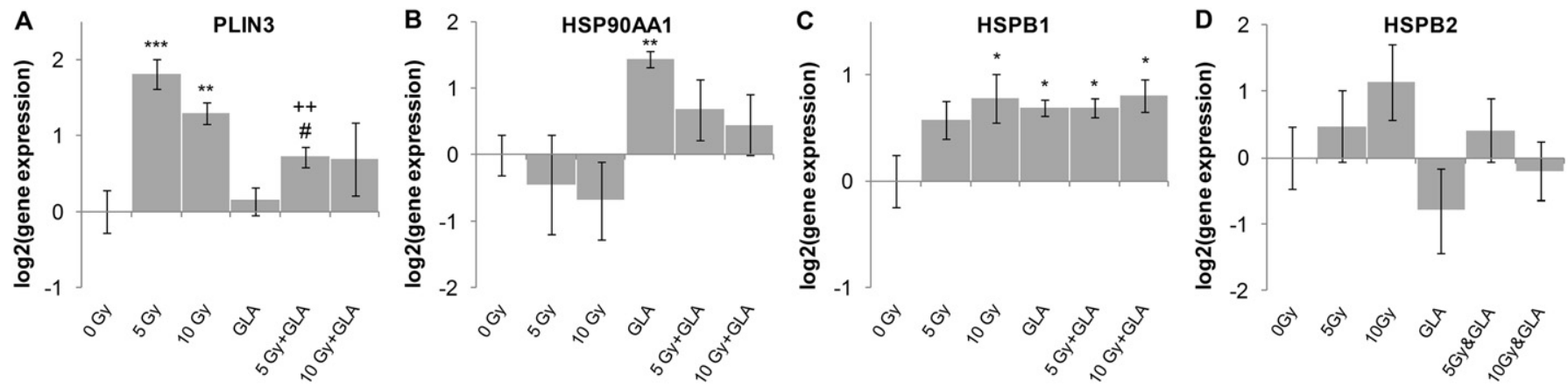

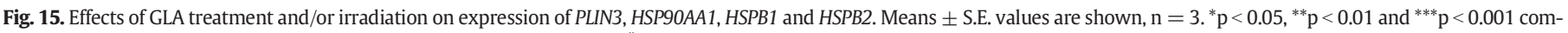
pared with control cells, ${ }^{++} \mathrm{p}<0.01$ compared with only irradiated cells, ${ }^{\#} \mathrm{p}<0.05$ compared with only GLA-treated cells.

(both 5 and $10 \mathrm{~Gy}$ ) significantly induced PLIN3 gene expression in U87 MG cells on incubation for $48 \mathrm{~h}$ (Fig. 15A). In contrast, it was previously reported that the expression of PLIN3 protein decreases by $40-60 \%$ due to the irradiation of U87 MG cells with 2 Gy [71]. Moreover, it seems that different PUFAs have differential effects on the expression of PLIN3. For example, PLIN3 was up-regulated by EPA treatment in goat monocytes, but not affected by DHA [71]. In our study GLA treatment did not alter the expression of PLIN3 in U87 MG cells, while when GLA was added as an adjuvant to irradiation, the over-expression of PLIN3 was normalized as compared with cells treated with irradiation alone. Confocal microscopy revealed a significant increase in the amount of LD in GLAtreated + irradiated cells as compared with irradiated control samples.

Further studies are needed to disclose the detailed mechanism and kinetics of GLA- and irradiation-induced alterations in LD formation and how these changes affect the sensitivity of cancer cells to apoptosis.

In summary, we detected specific changes in FA biosynthetic gene expression in glioma cells after GLA and/or irradiation treatment. The same samples yielded novel and detailed information on the FA and lipid profiles revealing specific alterations which differentiated each treatment protocol. The combination of two "omics" approaches allowed a functional explanation of enhanced tumor cell death. It is suggested that targeting the lipid composition by means of "membranelipid therapy" [72], e.g. by PUFA supplementation, may alter the radiosensitivity of gliomas. The increased PUFA content is coupled with PS oxidation and externalization and contributes to the induction of plasma membrane-regulated apoptosis. A summary of the main pathways of FA biosynthesis and how GLA treatment or irradiation influenced the expression of the corresponding genes is presented in Fig. 16.

Further experiments are needed to compare our in vitro data on gene expression and lipidome changes with in vivo results, and possibly clinical samples. This might promote the design of optimal irradiation protocols and possibly combination therapies involving the use of PUFAs.

\section{Methods}

\subsection{Materials}

Lipid standards were obtained from Avanti Polar Lipids (Alabaster, AL, USA). The solvents used for extraction and for MS analyses were of liquid chromatographic grade from Merck (Darmstadt, Germany) and Optima LC-MS grade from Thermo Fisher Scientific (Waltham, MA, USA). All other chemicals were purchased from Sigma and were of the best available grade.

\subsection{Cell culturing and treatment}

U87 MG (ATCC HTB-14 ${ }^{\mathrm{TM}}$ ) glioma cells were cultured at $37^{\circ} \mathrm{C}$ in $5 \%$ $\mathrm{CO} 2$ at atmospheric pressure, in DMEM supplemented with $10 \%$ FCS. 500,000 cells were plated for gene expression and LTQ Orbitrap analysis in 6-well plates (Corning Costar). 2000 cells/well were plated on 8-well Lab-Tek II chambered coverglass (Thermo Fisher Scientific) for confocal microscopic analysis. 40,000 cells/well were plated in 24-well TC flasks in $500 \mu \mathrm{L}$ medium for FACS analysis. Cells were incubated for $24 \mathrm{~h}$ before treatment. Cells were treated with GLA ester (Ubichem Research, Budapest, Hungary) at $50 \mu \mathrm{M}$. In the event of co-treatment with irradiation $1 \mathrm{~h}$ after GLA treatment cells were exposed to 5 or $10 \mathrm{~Gy}$ irradiation and then incubated for $48 \mathrm{~h}$. Irradiation was performed as described previously [4]. All measurements were carried out with three independent biological replicates.

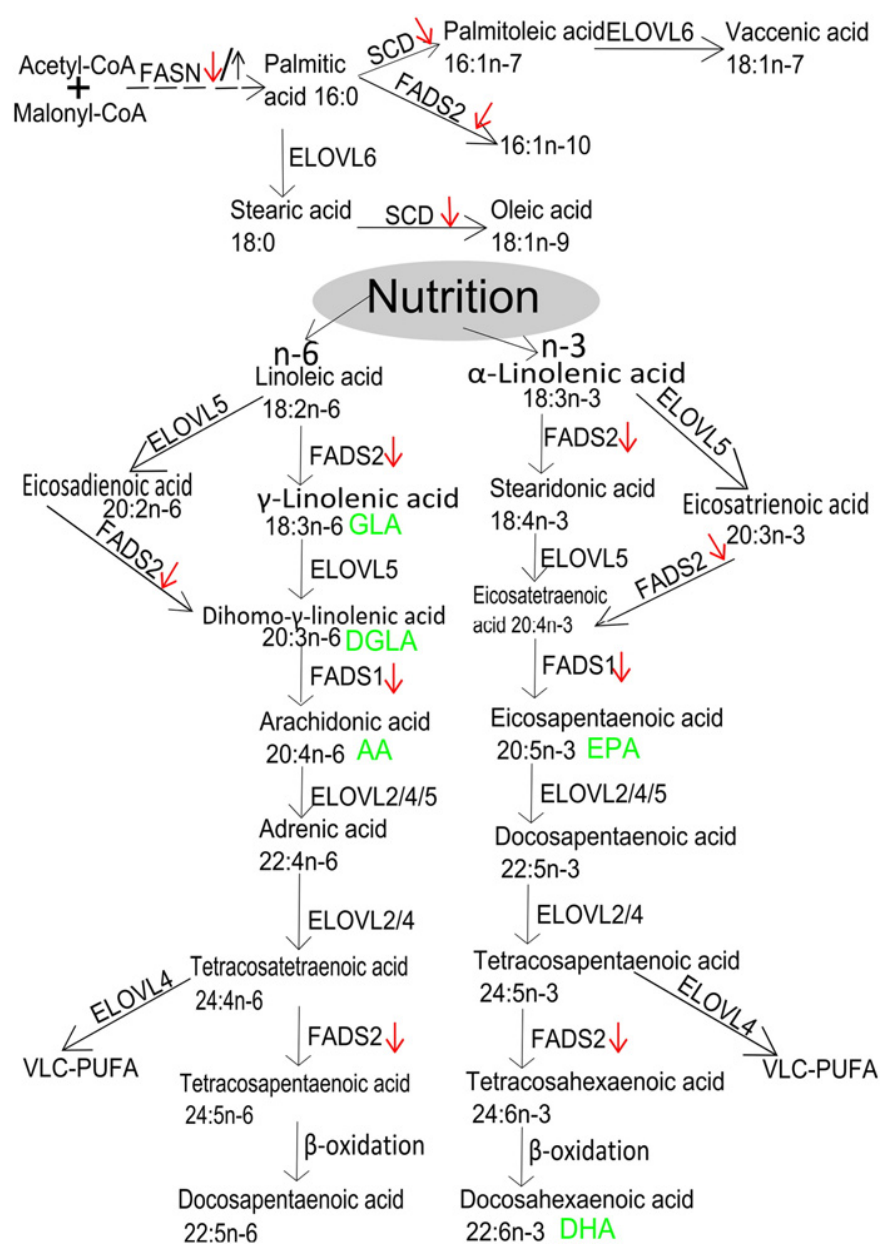

Fig. 16. SFA, MUFA and PUFA biosynthesis in humans. The scheme was prepared after $[32,52,73,74,75]$. The meanings of the symbols: $\downarrow$ : GLA treatment diminished the expression of the respective gene; $\uparrow:$ irradiation increased the expression of the respective gene. 


\subsection{RNA extraction and $R T-P C R$}

Total RNA was isolated with Accuzol ${ }^{\mathrm{TM}}$ Total RNA Extraction Solution (Bioneer, Daejon, South Korea), according to the manufacturer's instructions. The quality and quantity of RNA were determined with NanoDrop1000 Version 3.8.1. (Thermo Fisher Scientific, Wilmington, DE, USA). $1.5 \mu$ R RA was transcribed with the High Capacity cDNA Reverse Transcription Kit (Applied Biosystems ${ }^{\circledR}$, Life Technologies, Foster City, CA, USA). cDNA was diluted 8-fold. QRT-PCR was performed on a RotorGene 3000 instrument (Corbett Life Science, QIAGEN). Gene-specific primers and SybrGreen amplification protocol were used as described previously with slight modifications [76]. Supplementary Table $2 \mathrm{~S}$ shows the primer sequences used in the study. The reaction mix was prepared with $4.5 \mu \mathrm{L}$ cDNA, $0.5 \mu \mathrm{L}$ primer $(10 \mathrm{pM})$ and $5 \mu \mathrm{L}$ FastStart SYBR Green Master (Roche Diagnostics, USA). PCR was performed with 55 cycles of the following protocol: $1.95{ }^{\circ} \mathrm{C}$ for $10 \mathrm{~min} ; 2.95^{\circ} \mathrm{C}$ for $15 \mathrm{~s} ; 3.60^{\circ} \mathrm{C}$ for $10 \mathrm{~s}$; and $4.72{ }^{\circ} \mathrm{C}$ for $20 \mathrm{~s}$. Ct values were determined and amplification specificity was verified by melting curve analysis. Data were normalized to the average of two control genes (HPRT1 and PPIA). $\Delta \Delta \mathrm{Ct}$ values were calculated to compare the expression of treated cells with that of control cells. Significance was determined by using Welch's $t$-test.

\subsection{Evaluation of LD content with confocal microscopy}

To determine LD content, live cells were stained for 30 min with $25 \mu \mathrm{M}$ AC-202, a fluorescent LD-specific dye [21,77]. Olympus Fluoview FV1000 confocal laser scanning microscope (Olympus Life Science Europa GmbH, Hamburg, Germany) with a UPLSAPO 20× (dry, NA:0.75) objective was used to visualize U87 MG cells containing LDs. Eight-well LabTek II coverslip-bottom chambers (Thermo Fisher Scientific, Wilmington, DE, USA) were used for cell culturing and for each sample at least 12 microscope fields were analyzed in 4 different replicate wells. After 8-bit conversion, fluorescence images were thresholded by using Image J software. A fixed intensity range of 50-255 and "dark background" options were used during thresholding. The "set measurements" option of Image J was limited to "area measurement" and "limit to threshold". The percentage of the area occupied by LDs was calculated from the total cellular area (manual tracing of cell borders on transmission images using Olympus Fluoview software) and thresholded LD areas by using the "measure" function of Image J.

\subsection{FACS analysis}

U87 MG cells $(40,000)$ were plated in 24-well tissue culture plates (Corning Life Sciences) and were treated with GLA and/or irradiation. After $48 \mathrm{~h}$ the cells were collected, and resuspended in Annexin $\mathrm{V}$ binding buffer (0.01 M HEPES, $0.14 \mathrm{M} \mathrm{NaCl}$ and $2.5 \mathrm{mM} \mathrm{CaCl}_{2}$ ). Annexin V-Alexa 488 (Life Technologies, 2.5:100) and propidium iodide $(10 \mu \mathrm{g} / \mathrm{ml})$ (Sigma-Aldrich, Hungary) were added to the cells, which were then kept for $15 \mathrm{~min}$ in the dark at room temperature. After washing, the cells were analyzed on a FACSCalibur cytofluorimeter (Becton Dickinson). The percentage of the FL1 (AnnexinV-Alexa 488) positive and FL3 (propidium iodide) negative early apoptotic cells and FL1 (AnnexinV-Alexa 488) positive and FL3 (propidium iodide) positive late apoptotic cells were determined. For statistical significance two two-tailed Student's t-test was used.

\subsection{Lipid extraction}

After treatment, the cells were centrifuged, and the lipids were extracted according to a modified Folch procedure as described by Balogh et al. [64]. The lipid extract was reconstituted in $200 \mu \mathrm{L}$ chloroform:methanol 1:1 (by vol.). Lipid species were annotated according to the updated comprehensive classification system for lipids [78].

\subsection{Mass spectrometry}

MS analyses were performed on a LTQ-Orbitrap Elite instrument (Thermo Fisher Scientific, Bremen, Germany) equipped with a robotic nanoflow ion source TriVersa NanoMate (Advion BioSciences, Ithaca, $\mathrm{NY}$ ), using chips with the diameter of 5.5-mm spraying nozzles. The ion source was controlled by Chipsoft 8.3.1 software. The ionization voltages were $+1.3 \mathrm{kV}$ and $-1.9 \mathrm{kV}$ in positive and negative mode, respectively, and the back-pressure was set at $1 \mathrm{psi}$ in both modes. The temperature of the ion transfer capillary was $250{ }^{\circ} \mathrm{C}$.

For quantification, $5 \mu \mathrm{L}$ of lipid extract was spiked before the MS measurement with an internal standard mix containing $114 \mathrm{pmol}$ PC D31-16:0/18:1, 40 pmol PE D31-16:0/18:1, 17 pmol PI D31-16:0/18:1, 30 pmol PS D31-16:0/18:1, 4 pmol PG D31-16:0/18:1, 1 pmol PA D3116:0/18:1, 2 pmol CL 56:0, 3 pmol Cer d18:1/17:0, 5 pmol GluCer d18:1/12:0, 8 pmol SM d18:1/17:0, and 2 pmol TAG 51:3. Each spiked extract was diluted with $300 \mu \mathrm{L}$ chloroform:methanol 1:3 (by vol.), and then divided into two parts, and 5\% dimethylformamide (as an additive in the negative ion mode) or $7.5 \mathrm{mM}$ ammonium chloride (as an additive in the positive ion mode) was added to the halves. The lipid classes: PC (diacyl), PC-O (alkyl-acyl), LPC, SM, TAG and CholE were detected and quantified in the positive ion mode, and PE (diacyl), PE-Pl (alkenyl-acyl), PI, PG, CL, PA, PS, Cer and GluCer in the negative ion mode. Each of the quantified lipid species accounted for more than $0.5 \%$ within its lipid class.

Lipids were identified by LipidXplorer software [79] by matching the $\mathrm{m} / \mathrm{z}$ values of their monoisotopic peaks to the corresponding elemental composition constraints. The mass tolerance was $2 \mathrm{ppm}$ and the intensity threshold was set according to the noise level reported by Xcalibur software (Thermo Scientific). Statistical analyses of the lipidomic datasets were performed with MetaboAnalyst [80], according to Storey and Tibshirani [81] or to Benjamini and Hochberg [82], as indicated.

Total FA analysis of the lipid extracts was carried out with a GC-MS system (GCMS-QP2010, Shimadzu, Japan) equipped with a BPX-70 capillary column ( $10 \mathrm{~m} \times 0.1 \mathrm{~mm}$ ID, $0.2 \mu \mathrm{m}$ film thickness) as described by Balogh et al. [72].

Supplementary data to this article can be found online at http://dx. doi.org/10.1016/j.bbalip.2015.06.003.

$\begin{array}{ll}\text { Abbreviations } \\ \text { AA } & \text { arachidonic acid } \\ \text { Cer } & \begin{array}{l}\text { ceramide } \\ \text { CholE }\end{array} \\ \text { cholesteryl ester } \\ \text { CL } & \text { cardiolipin } \\ \text { DGLA } & \text { dihomo-gamma-linolenic acid } \\ \text { DHA } & \text { docosahexaenoic acid } \\ \text { EPA } & \text { eicosapentaenoic acid } \\ \text { FA } & \text { fatty acid } \\ \text { GLA } & \text { gamma-linolenic acid } \\ \text { GICer } & \text { glucosylceramide } \\ \text { HSP } & \text { heat shock protein } \\ \text { LD } & \text { lipid droplet } \\ \text { LPC } & \text { lysophosphatidylcholine } \\ \text { MUFA } & \text { monounsaturated fatty acid } \\ \text { PC } & \text { phosphatidylcholine } \\ \text { PE } & \text { phosphatidylethanolamine } \\ \text { PI } & \text { phosphatidylinositol } \\ \text { PG } & \text { phosphatidylglycerol } \\ \text { PS } & \text { phosphatidylserine } \\ \text { PUFA } & \text { polyunsaturated fatty acid } \\ \text { ROS } & \text { reactive oxygen species } \\ \text { SM } & \text { sphingomyelin } \\ \text { SFA } & \text { saturated fatty acid } \\ \text { TAG } & \text { triacylglycerol }\end{array}$




\section{Competing interests}

The authors declare that no competing interest exists.

\section{Author contributions}

The study was planned by LGP, LH, KK, LV and OA. Irradiation was carried out by LH, IM and KH, RNA isolation and gene expression analysis by OA, Lipidomic analyses by MP, LV and GB. Confocal microscopic images were recorded by FA. FACS measurements were made by GSZ and OA. LGP, LH and OA prepared the manuscript.

\section{Transparency document}

The Transparency document associated with this article can be found, in online version.

\section{Acknowledgments}

This study was supported by grants from the Hungarian Basic Research Fund (OTKA NK100857, NN111006), the New Szechenyi Plan (GOP-1.1.1-11-2012-0452) and the ELI-ALPS project (GOP-1.1.112/B-2012-0001), supported by the European Union and co-financed by the European Regional Development Fund.

\section{References}

[1] U.N. Das, W. Prasad, D.R. Reddy, Local application of $\gamma$-linolenic acid in the treatment of human glioma, Cancer Lett. 94 (2) (1995) 147-155.

[2] U.N. Das, From bench to the clinic: gamma-linolenic acid therapy of human gliomas, Prostaglandins Leukot. Essent. Fat. Acids 70 (6) (2004) 539-552.

[3] U.N. Das, Gamma-linolenic acid therapy of human glioma-a review of in vitro, in vivo, and clinical studies, Med. Sci. Monit. 13 (7) (2007) RA119-RA131.

[4] O. Antal, L. Hackler, J. Shen, I. Mán, K. Hideghéty, K. Kitajka, et al., Combination of unsaturated fatty acids and ionizing radiation on human glioma cells: cellular, biochemical and gene expression analysis, Lipids Health Dis. 13 (2014) 142.

[5] N. Faragó, L.Z. Fehér, K. Kitajka, U.N. Das, L.G. Puskás, MicroRNA profile of polyunsaturated fatty acid treated glioma cells reveal apoptosis-specific expression changes, Lipids Health Dis. 10 (2011) 173.

[6] H.A. Leaver, H.S. Bell, M.T. Rizzo, J.W. Ironside, A. Gregor, S.B. Wharton, et al. Antitumour and pro-apoptotic actions of highly unsaturated fatty acids in glioma, Prostaglandins Leukot. Essent. Fat. Acids 66 (2002) 19-29.

[7] M. Preuss, G.D. Girnun, C.J. Darby, N. Khoo, A.A. Spector, M.E. Robbins, Role of antioxidant enzyme expression in the selective cytotoxic response of glioma cells to gamma-linolenic acid supplementation, Free Radic. Biol. Med. 28 (2000) 1143-1156.

[8] S.S. Sandrone, G. Repossi, M. Candolfi, A.R. Eynard, Polyunsaturated fatty acids and gliomas: A critical review of experimental, clinical, and epidemiologic data, Nutrition 30 (2014) 1104-1109.

[9] S. Vartak, R. McCaw, C.S. Davis, M.E. Robbins, A.A. Spector, Gamma-linolenic acid (GLA) is cytotoxic to 36B10 malignant rat astrocytoma cells but not to 'normal' rat astrocytes, Br. J. Cancer 77 (1998) 1612-1620.

[10] S. Vartak, M.E. Robbins, A.A. Spector, Polyunsaturated fatty acids increase the sensitivity of 36B10 rat astrocytoma cells to radiation-induced cell kill, Lipids 32 (1997) 283-292.

[11] X. Lu, H. Yu, Q. Ma, S. Shen, U.N. Das, Linoleic acid suppresses colorectal cancer cell growth by inducing oxidant stress and mitochondrial dysfunction, Lipids Health Dis. 9 (2010) 106

[12] S. Colas, L. Paon, F. Denis, M. Prat, P. Louisot, C. Hoinard, et al., Enhanced radiosensitivity of rat autochthonous mammary tumor by dietary docosahexaenoic acid, Int. J. Cancer 109 (2004) 449-454.

[13] Y.M. Dupertuis, M.M. Mequid, C. Pichard, Colon cancer therapy: new perspectives of nutritional manipulations using polyunsaturated fatty acids, Curr. Opin. Clin. Nutr. Metab. Care. 10 (2007) 427-432.

[14] Y. Harasaki, A. Waziri, Potential usefulness of radiosensitizers in glioblastoma, Neurosurg. Clin. N. Am. 3 (2012) 429-437.

[15] M. Péter, G. Balogh, I. Gombos, G. Liebisch, I. Horváth, Z. Török, et al., Nutritional lipid supply can control the heat shock response of B16 melanoma cells in culture, Mol. Membr. Biol. 29 (2012) 274-289.

[16] P.V. Escribá, J.M. González-Ros, F.M. Goñi, P.K. Kinnunen, L. Vigh, L. SánchezMagraner, et al., Membranes: a meeting point for lipids, proteins and therapies, J. Cell. Mol. Med. 12 (2008) 829-875.

[17] V. Chajès, M. Cambot, K. Moreau, G.M. Lenoir, V. Joulin, Acetyl-CoA carboxylase alpha is essential to breast cancer cell survival, Cancer Res. 66 (2006) 5287-5294.

[18] Hatzivassiliou G1, F. Zhao, D.E. Bauer, C. Andreadis, A.N. Shaw, et al., ATP citrate lyase inhibition can suppress tumor cell growth, Cancer Cell 8 (2005) 311-321.
[19] R.A. Igal, Stearoyl-CoA desaturase-1: a novel key player in the mechanisms of cell proliferation, programmed cell death and transformation to cancer, Carcinogenesis 31 (2010) 1509-1515.

[20] D.W. Bilheimer, L.M. Buja, R.W. Parkey, F.J. Bonte, J.T. Willerson, Fatty acid accumulation and abnormal lipid deposition in peripheral and border zones of experimental myocardial infarcts, J. Nucl. Med. 19 (3) (1978) 276-283.

[21] L.G. Puskás, L.Z. Fehér, C. Vizler, F. Ayaydin, E. Rásó, E. Molnár, et al., Polyunsaturated fatty acids synergize with lipid droplet binding thalidomide analogs to induce oxidative stress in cancer cells, Lipids Health Dis. 9 (2010) 56.

[22] L.I. Nagy, E. Molnár, I. Kanizsi, R. Madácsi, B. Ózsvári, L.Z. Fehér, et al., Lipid droplet binding thalidomide analogs activate endoplasmic reticulum stress and suppress hepatocellular carcinoma in a chemically induced transgenic mice model, Lipids Health Dis. 12 (2013) 175.

[23] B. Wen, E. Deutsch, P. Opolon, A. Auperin, V. Frascogna, E. Connault, et al., N-3 polyunsaturated fatty acids decrease mucosal/epidermal reactions and enhance antitumour effect of ionising radiation with inhibition of tumour angiogenesis, $\mathrm{Br}$. J. Cancer 89 (2003) 1102-1107.

[24] B. Bucci, S. Misiti, A. Cannizzaro, R. Marchese, G.H. Raza, R. Miceli, et al., Fractionated ionizing radiation exposure induces apoptosis through caspase- 3 activation and reactive oxygen species generation, Anticancer Res. 26 (2006) 4549-4557.

[25] A. Jakobsson, R. Westerberg, A. Jacobsson, Fatty acid elongases in mammals: their regulation and roles in metabolism, Prog. Lipid Res. 45 (3) (2006) 237-249.

[26] F.P. Kuhajda, Fatty acid synthase and cancer: new application of an old pathway, Cancer Res. 66 (12) (2006) 5977-5980.

[27] J.L. Little, F.B. Wheeler, D.R. Fels, C. Koumenis, S.J. Kridel, Inhibition of fatty acid synthase induces endoplasmic reticulum stress in tumor cells, Cancer Res. 67 (2007) 1262-1269.

[28] K.E. Hopperton, R.E. Duncan, R.P. Bazinet, M.C. Archer, Fatty acid synthase plays a role in cancer metabolism beyond providing fatty acids for phospholipid synthesis or sustaining elevations in glycolytic activity, Exp. Cell Res. 320 (2014) 302-310.

[29] J.A. Menendez, R. Lupu, Fatty acid synthase and the lipogenic phenotype in cancer pathogenesis, Nat. Rev. Cancer 7 (2007) 763-777.

[30] D.K. Nomura, J.Z. Long, S. Niessen, H.S. Hoover, S.W. Ng, B.F. Cravatt, Monoacylglycerol lipase regulates a fatty acid network that promotes cancer pathogenesis, Cell 140 (2010) 49-61.

[31] H. Lee, W.J. Park, Unsaturated Fatty Acids, Desaturases, and Human Health, J. Med. Food 17 (2014) 189-197.

[32] D.I. Sinner, G.J. Kim, G.C. Henderson, R.A. Igal, StearoylCoA desaturase-5: a novel regulator of neuronal cell proliferation and differentiation, PLoS One 7 (2012) e39787.

[33] N. Scaglia, R.A. Igal, Stearoyl-CoA desaturase is involved in the control of proliferation, anchorage-independent growth, and survival in human transformed cells, J. Biol. Chem. 280 (2005) 25339-25349.

[34] N. Scaglia, R.A. Igal, Inhibition of Stearoyl-CoA Desaturase 1 expression in human lung adenocarcinomacells impairs tumorigenesis, Int. J. Oncol. 33 (4) (2008) 839-850.

[35] Y.Z. Chen, J.Y. Xue, C.M. Chen, B.L. Yang, Q.H. Xu, F. Wu, et al., PPAR signaling pathway may be an important predictor of breast cancer response to neoadjuvant chemotherapy, Cancer Chemother. Pharmacol. 70 (2012) 637-644.

[36] J. Nasrollahzadeh, F. Siassi, M. Doosti, M.R. Eshraghian, F. Shokri, M.H. Modarressi, et al., The influence of feeding linoleic, gamma linolenic and docosahexaenoic acid rich oils on rat brain tumor fatty acids composition on fatty acid binding protein 7 mRNA expression, Lipids Health Dis. 7 (2008) 45

[37] K.L. Ramos, A. Colquhoun, Protective role of glucose-6-phosphate dehydrogenase activity in the metabolic response of $\mathrm{C} 6$ rat glioma cells to polyunsaturated fatty acid exposure, Glia 43 (2003) 149-166.

[38] F. Fujiwara, S. Todo, S. Imashuku, Fatty acid modification of cultured neuroblastoma cells by gamma linolenic acid relevant to its antitumor effect, Prostaglandins Leukot. Med. 30 (1987) 37-49.

[39] D.B. Jump, S.D. Clarke, A. Thelen, M. Liimatta, Coordinate regulation of glycolytic and lipogenic gene expression by polyunsaturated fatty acids, J. Lipid Res. 35 (1994) 1076-1084.

[40] M.K. Mater, A.P. Thelen, D.A. Pan, D.B. Jump, Sterol response element- binding protein 1c (SREBP1c) is involved in the polyunsaturated fatty acid suppression of hepatic S14 gene transcription, J. Biol. Chem. 274 (46) (1999) 32725-32732.

[41] W.J. Park, K.S. Kothpalli, P. Lawrence, J.T. Brenna, FADS2 function loss at the cancer hotspot 11q13 locus diverts lipid signaling precursor synthesis to unusual eicosanoid fatty acids, PLoS One 6 (2011) e28186.

[42] R. Portolesi, B.C. Powel, R.A. Gibson, Delta6 desaturase mRNA abundance in HepG2 cells is suppressed by unsaturated fatty acids, Lipids 43 (2008) 91-95.

[43] T.Y. Nara, W.S. He, C. Tang, S.D. Clarke, M.T. Nakamura, The E-box like sterol regulatory element mediates the suppression of human D-6 desaturase gene by highly unsaturated fatty acids, Biochem. Biophys. Res. Commun. 296 (2002) 111-117.

[44] B. Vessby, I.B. Gustafsson, S. Tengblad, M. Boberg, A. Andersson, Desaturation and elongation of fatty acids and insulin action, Ann. N. Y. Acad. Sci. 967 (2002) 183-195.

[45] H.T. Reardon, A.T. Hsieh, W.J. Park, K.S. Kothapalli, J.C. Anthony, P.W. Nathanielsz, et al., Dietary long-chain polyunsaturated fatty acids upregulate expression of FADS3 transcripts, Prostaglandins Leukot. Essent. Fat. Acids 88 (2013) 15-19.

[46] J.M. Ntambi, Regulation of stearoyl-CoA desaturase by polyunsaturated fatty acids and cholesterol, J. Lipid Res. 40 (1999) 1549-1558.

[47] T. Matsuzaka, H. Shimano, N. Yahagi, M. Amemiya-Kudo, T. Yoshikawa, A.H. Hasty, et al., Dual regulation of mouse Delta(5)- and Delta(6)-desaturase gene expression by SREBP-1 and PPARalpha, J. Lipid Res. 43 (2002) 107-114.

[48] H. Blanchard, P. Legrand, F. Pédrono, Fatty Acid Desaturase 3 (Fads3) is a singular member of the Fads cluster, Biochimie 93 (2011) 87-90. 
[49] V. Wijendran, I. Downs, C.T. Srigley, K.S. Kothpalli, W.J. Park, B.S. Blank, et al., Dietary arachidonic acid and docosahexaenoic acid regulate liver fatty acid desaturase (FADS) alternative transcript expression in suckling piglets, Prostaglandins Leukot. Essent. Fat. Acids 89 (2013) 345-350.

[50] C.R. Santos, A. Schulze, Lipid metabolism in cancer, FEBS J. 279 (2012) 2610-2623.

[51] A.E. Leonard, S.L. Pereira, H. Sprecher, Y.S. Huang, Elongation of long-chain fatty acids, Prog. Lipid Res. 43 (2004) 36-54.

[52] Y. Ohno, S. Suto, M. Yamanaka, Y. Mizutani, S. Mitsutake, Y. Igarashi, et al., ELOVL1 production of C24 acyl-CoAs is linked to C24 sphingolipid synthesis, Proc. Natl. Acad. Sci. U. S. A. 107 (2010) 18439-18444.

[53] A. Kihara, Very long-chain fatty acids: elongation, physiology and related disorders, J. Biochem. 152 (2012) 387-395.

[54] G. Balogh, M. Péter, A. Glatz, I. Gombos, Z. Török, I. Horváth, et al., Key role of lipids in heat stress management, FEBS Lett. 587 (2013) 1970-1980.

[55] J. Fu, D. Koul, J. Yao, S. Wang, Y. Yuan, H. Colman, et al., Novel HSP90 inhibitor NVPHSP990 targets cell cycle regulators to ablate Olig2-positive glioma tumor initiating cells, Cancer Res. 73 (2013) 3062-3074.

[56] E.I. Azzam, J.P. Jay-Gerin, D. Pain, Ionizing radiation-induced metabolic oxidative stress and prolonged cell injury, Cancer Lett. 327 (2012) 48-60.

[57] Tyurina YY1, V.A. Tyurin, V.I. Kapralova, K. Wasserloos, M. Mosher, M.W. Epperly, J.S. Greenberger, B.R. Pitt, V.E. Kagan, Oxidative lipidomics of $\gamma$-radiation-induced lung injury: mass spectrometric characterization of cardiolipin and phosphatidylserine peroxidation, Radiat. Res. 175 (2011) 610-621.

[58] M.G. Salgo, F.P. Corongiu, A. Sevanian, Enhanced interfacial catalysis and hydrolytic specificity of phospholipase A2 toward peroxidized phosphatidylcholine vesicles, Arch. Biochem. Biophys. 304 (1993) 123-132.

[59] C. Code, A.K. Mahalka, K. Bry, P.K. Kinnunen, Activation of phospholipase A2 by 1 palmitoyl-2-(9'-oxo-nonanoyl)-sn-glycero-3-phosphocholine in vitro, Biochim. Biophys. Acta 2010 (1798) 1593-1600.

[60] A. Laszlo, D. Thotala, D.E. Hallahan, Membrane phospholipids, EML4-ALK, and Hsp90 as novel targets in lung cancer treatment, Cancer J. 19 (2013) 238-246.

[61] V.A. Tyurin, Y.Y. Tyurina, V.B. Ritov, A. Lysytsya, A.A. Amoscato, P.M. Kochanek, et al., Oxidative lipidomics of apoptosis: quantitative assessment of phospholipid hydroperoxides in cells and tissues, Methods Mol. Biol. 610 (2010) 353-374.

[62] L. Vigh, B. Maresca, J.L. Harword, Does the membrane's physical state control the expression of heat shock and other genes? Trends Biochem. Sci. 23 (1998) 369-374.

[63] G. Balogh, M. Péter, G. Liebisch, I. Horváth, Z. Török, E. Nagy, et al., Lipidomics reveals membrane lipid remodelling and release of potential lipid mediators during early stress responses in a murine melanoma cell line, Biochim. Biophys. Acta 2010 (1801) 1036-1047.

[64] A.T. Jacobs, L.J. Mamett, Heat shock factor 1 attenuates 4-Hydroxynonenal-mediated apoptosis: critical role for heat shock protein 70 induction and stabilization of BclXL, J. Biol. Chem. 282 (2007) 33412-33420.

[65] Z. Fuks, R. Kolesnick, Engaging the vascular component of the tumor response, Cancer Cell 8 (2005) 89-91.
[66] I. Corre, M. Guillonneau, F. Paris, Membrane Signaling Induced by High Doses of Ionizing Radiation in the Endothelial Compartment, Int. J. Mol. Sci. 14 (2013) 22678-22696.

[67] Y. Song, L.J. Zhang, H. Li, Y. Gu, F.F. Li, L.N. Jiang, et al., Polyunsaturated fatty acid relatively decreases cholesterol content in THP-1 macrophage-derived foam cell: partly correlates with expression profile of CIDE and PAT members, Lipids Health Dis. 12 (2013) 111.

[68] A.S. Greenberg, R.A. Coleman, F.B. Kraemer, J.L. McManaman, M.S. Obin, V. Puri, et al. The role of lipid droplets in metabolic disease in rodents and humans, J. Clin. Invest. 121 (2011) 2102-2110.

[69] C. Sztalryd, A.R. Kimmel, Perilipins: lipid droplet coat proteins adapted for tissuespecific energy storage and utilization, and lipid cytoprotection, Biochimie 96 (2014) 96-101.

[70] C. Lecchi, G. Invernizzi, A. Agazzi, S. Modina, P. Sartorelli, G. Savoini, et al., Effects of EPA and DHA on lipid droplet accumulation and mRNA abundance of PAT proteins in caprine monocytes, Res. Vet. Sci. 94 (2013) 246-251.

[71] L. Vigh, I. Horváth, B. Maresca, J.L. Harwood, Can the stress protein response be controlled by 'membrane-lipid therapy'? Trends Biochem. Sci. 32 (2007) 357-363.

[72] L.F. Castro, Ó. Monroig, M.J. Leaver, J. Wilson, I. Cunha, D.R. Tocher, Functional desaturase Fads1 (D5) and Fads2 (D6) orthologues evolved before the origin of jawed vertebrates, PLoS One 7 (2012) e31950.

[73] M.K. Gregory, R.A. Gibson, R.J. Cook-Johnson, L.G. Cleland, M.J. James, Elongase reactions as control points in long-chain polyunsaturated fatty acid synthesis, PLoS One 6 (2011) e29662.

[74] KEGG: Kyoto Encyclopedia of Genes and Genomes, http://www.genome.jp/kegg/.

[75] A. Catalá, A. Zvara, L.G. Puskás, K. Kitajka, Melatonin-induced gene expression and its preventive effects on adriamycin-induced lipid peroxidation in rat liver, J. Pineal Res. 42 (2007) 43-49.

[76] S. Kuntam, L.G. Puskás, F. Ayaydin, Characterization of a new class of blue-fluorescent lipid droplet markers for live-cell imaging in plants, Plant Cell Rep. (2015).

[77] E. Fahy, S. Subramaniam, R.C. Murphy, M. Nishijima, C.R. Raetz, T. Shimizu, et al., Up date of the LIPID MAPS comprehensive classification system for lipids, J. Lipid Res. 50 (Suppl. S9-14) (2009).

[78] R. Herzog, D. Schwudke, K. Schuhmann, J.L. Sampaio, S.R. Bornstein, M. Schroeder, et al., A novel informatics concept for high-throughput shotgun lipidomics based on the molecular fragmentation query language, Genome Biol. 12 (2011) R8.

[79] J. Xia, Wishart. Web-based inference of biological patterns, functions and pathways from metabolomic data using MetaboAnalyst, Nat. Protoc. 6 (2011) 743-760.

[80] Tibshirani R. Storey, Statistical significance for genome wide studies, Proc. Natl Acad. Sci. U. S. A. 100 (2003) 9440-9445.

[81] Y. Benjamini, Y. Hochberg, Controlling the False Discovery Rate: A practical and powerful approach to multiple testing, J. R. Stat. Soc. B 57 (1995) 289-300.

[82] Y. Benjamini, Y. Hochberg, Controlling the False Discovery Rate: A practical and powerful approach to multiple testing, Journal of the Royal Statistical Society B 57 (1995) 289-300. 